\title{
A Subspace Blind Channel Estimation Method for OFDM Systems Without Cyclic Prefix
}

\author{
Sumit Roy, Senior Member, IEEE and Chengyang Li
}

\begin{abstract}
We propose a subspace based blind channel estimation method for orthogonal frequency-division multiplexing (OFDM) systems over a time-dispersive channel. Our approach is motivated by the resemblance of the multichannel signal model resulting from oversampling (or use of multiple receive sensors) of the received OFDM signal to that in conventional single carrier system. The proposed algorithm distinguishes itself from many previously reported channel estimation methods by the elimination of the cyclic prefix, thereby leading to higher channel utilization. Comparison of the proposed method with other two reported subspace channel estimation methods is presented by computer simulations to support its effectiveness.
\end{abstract}

Index Terms-Blind channel estimation, orthogonal frequencydivision multiplexing, oversampling, receiver diversity, subspace approach.

\section{INTRODUCTION}

$\mathbf{I}$ NTEREST in orthogonal frequency-division multiplexing [4], [5] (OFDM) has witnessed a rebirth in the context of next generation high-speed wireless/mobile communications systems due to its many advantages - notably, its high spectral efficiency, robustness to frequency selective fading, as well as the feasibility of low-cost transceiver implementations. Other than the discrete multitone (DMT) [6] architecture now standardized for xDSL applications, OFDM is being used for high-speed wireless LANs [7] (target rates 6-54 Mb/s) as well as wireless local loop applications in MMDS bands (1-10 Mb/s).

Channel estimation is indispensable to achieve coherent demodulation and consequently higher data rates. In practical OFDM systems operating over a time-dispersive channel, a cyclic prefix (CP) (or guard interval) longer than the channel duration is usually inserted in the transmitted sequence. As a result, the linear filtering by the channel is converted into a (complex) multiplicative distortion on each OFDM subchannel in the frequency domain. Appropriate training based approaches suffice to estimate the channel gains on each subchannel as described in [8]-[10]; the estimate can be then used for gain/phase correction [4], [5]. The length

\footnotetext{
Manuscript received June 26, 2000; revised April 8, 2001 and August 1, 2001; accepted August 16, 2001. The editor coordinating the review of this paper and approving it for publication is D. L. Goeckel. This work was supported in part by the Air Force Office of Scientific Research (AFOSR) under Grant F49620-1-0472 and in part by the National Science Foundation (NSF)/ITR under Grant CCR-0086032. This paper was presented at the Vehicular Technology Conference (Fall) 2001, Atlantic City, NJ, October 2001.

The authors are with the Department of Electrical Engineering, University of Washington, Seattle, WA 98195-2500 USA (e-mail: roy@ee.washington.edu; cyli@ee.washington.edu).

Digital Object Identifier 10.1109/TWC.2002.804160
}

of the $\mathrm{CP}$ is chosen for the maximum anticipated multipath spread; for IEEE 802.11a standard, this is $25 \%$ of an OFDM symbol duration, indicating a significant loss in utilization. Additionally, due to the time-varying nature of the channel, the training sequence needs to be transmitted.

The disadvantage of the aforementioned training based channel estimation methods naturally stimulates the search for blind channel estimation methods that avoid the use of the training sequence or even the CP. Recently, the presence of the $\mathrm{CP}$ has been exploited for blind channel estimation based on second order statistics [2], [3]. These methods use the channel output sequence prior to the CP removal and subsequent FFT operation. Specifically, Heath and Giannakis [2] proposed a spectrum fitting blind method based on the cyclostationarity property of the autocorrelation of the received data samples due to the $\mathrm{CP}$ insertion at the transmitter; this method, however, suffers from slow convergence of the estimator. Most recently, Cai and Akansu [3] developed a noise subspace method by utilizing the structure of the filtering matrix introduced by the $\mathrm{CP}$ insertion; it achieves faster convergence for smaller data records.

The main contribution of this paper is a blind subspace channel estimation algorithm which avoids the use of the $C P$ (thus, improving channel utilization) while achieving performance comparable to [3] with regards to estimator accuracy and convergence speed. However, the method requires oversampling or receiver diversity, thereby increasing receiver cost/complexity. Nonetheless, typical oversampling factors of $M=2$ are expected to be reasonable for implementation. Also, minimum mean-squared error (receiver) diversity combining has already been suggested to improve detection performance in [11] subsequent to channel estimation.

The rest of the paper is organized as follows. A baseband multichannel signal model for the OFDM system is introduced in Section II. The subspace based channel estimator is developed in Section III, where a sufficient condition on channel identifiability adjusted from [1] is described as well as a first-order performance analysis on the estimator. Computer simulations are conducted in Section IV to demonstrate the performance of the proposed algorithm with comparison to the two reported subspace methods [2] and [3]. Finally, Section V concludes the paper.

The notation used in this paper follows usual convention-vectors are denoted by symbols in boldface, $(\cdot)^{*},(\cdot)^{T},(\cdot)^{H}$ are complex conjugate, transpose and conjugate transpose of $(\cdot)$, respectively. $E(\cdot)$ is the mathematical expectation of $(\cdot) \cdot \operatorname{ran}(\cdot)$ and $\|\cdot\|$ give, respectively, the range and Frobenius norm of the matrix argument. 


\section{SignAl Formulation}

In this section, we describe a multichannel signal model for an OFDM system resulting from oversampling or multiple receiving sensors which closely resembles the model for single carrier system as in [1]. Consider an OFDM system as in Fig. 1 with $Q$ subcarriers and no cyclic prefix extensions. The $k$ th block of the "frequency domain" information symbols is

$$
\mathbf{s}(k)=\left[s_{0}(k), s_{1}(k), \ldots, s_{Q-1}(k)\right]^{T} .
$$

For information symbol duration of $T$, the corresponding OFDM symbol duration $T_{s}=Q T$. After multicarrier modulation implemented by IFFT, the "time domain" output signal vector is given by

$$
\mathbf{x}(k)=\left[x_{0}(k), x_{1}(k), \ldots, x_{Q-1}(k)\right]^{T}=\mathbf{W}_{Q} \mathbf{s}(k)
$$

where $\mathbf{W}_{Q}$ is the $Q \times Q$-dimensional IDFT matrix

$$
\mathbf{W}_{Q}=\frac{1}{\sqrt{Q}}\left[\begin{array}{cccc}
1 & 1 & \cdots & 1 \\
1 & W_{Q}^{-1} & \cdots & W_{Q}^{-(Q-1)} \\
1 & W_{Q}^{-2} & \cdots & W_{Q}^{-2(Q-1)} \\
\vdots & & & \vdots \\
1 & W_{Q}^{-(Q-1)} & \ldots & W_{Q}^{-(Q-1)(Q-1)}
\end{array}\right]
$$

with $W_{Q}=e^{-j 2 \pi / Q}$.

Each element of $\mathbf{x}(k)$ is then pulse shaped by $g_{\mathrm{tr}}(t)$ to generate the continuous time signal sent on the channel

$$
x(t)=\sum_{k=-\infty}^{+\infty} \sum_{p=0}^{Q-1} x_{p}(k) g_{\mathrm{tr}}\left(t-p T-k T_{s}\right)
$$

\section{A. Multichannel Model for Oversampling}

Substituting $T_{s}=Q T$ in (3) leads to

$$
x(t)=\sum_{k=-\infty}^{+\infty} \sum_{p=0}^{Q-1} x_{p}(k) g_{\mathrm{tr}}(t-(p+k Q) T)
$$

Thus, denoting another subscript index $q=p+k Q$, we identify $k=\lfloor(q / Q)\rfloor(\lfloor x\rfloor$ is the largest integer contained in $x)$ and $p=$ $q$ modulo $Q$. Then the transmitted signal $x(t)$ can be rewritten as

$$
x(t)=\sum_{q=-\infty}^{+\infty} x_{q} g_{\mathrm{tr}}(t-q T)
$$

The signal $x(t)$ passes through a dispersive channel with impulse response $c(t)$ and is contaminated by additive white Gaussian noise (AWGN) $n(t)$, and is input into a front-end receive filter $g_{r x}(t)$.

Defining the composite channel filter $h(t)=g_{t r}(t) * c(t) *$ $g_{r x}(t)$ and the filtered noise $v(t)=n(t) * g_{r x}(t)$ where $*$ denotes linear convolution, the received signal $r(t)$ is, therefore

$$
r(t)=\sum_{q=-\infty}^{+\infty} x_{q} h(t-q T)+v(t) .
$$

Assume the composite channel $h(t)$ to have finite support $[0,(L+1) T)$ no longer than the OFDM symbol duration $T_{s}$; this implies that any intersymbol interference (ISI) is only restricted to the past neighboring symbol as is generally true for OFDM. A synchronized rate $M / T$ sampler (i.e., oversampling factor of $M$ compared with information symbol sampling rate $1 / T$ ) after $r(t)$ yields (for $m=0, \ldots, M-1$ )

$$
\begin{aligned}
r_{i}^{(m)} & =r\left(t_{0}+i T+\frac{m T}{M}\right) \\
& =\sum_{l=0}^{L} x_{i-l} h\left(t_{0}+l T+\frac{m T}{M}\right)+v_{i}^{(m)}
\end{aligned}
$$

where $v_{i}^{(m)}=v\left(t_{0}+i T+(m T / M)\right)$.

Define $h^{(m)}(l)=h\left(t_{0}+l T+(m T / M)\right)$ and

$$
\mathbf{h}^{(m)}=\left[h^{(m)}(0), h^{(m)}(1), \ldots, h^{(m)}(L)\right]^{T} .
$$

As mentioned earlier, the (finite support) dispersive channel causes the output $\mathbf{x}(k-1)$ corresponding to the $(k-1)$ th OFDM symbol to partly overlap with the output for the $k$ th symbol $\mathbf{x}(k)$; the ISI affects the beginning $M L$ samples among the $M Q$ samples in the duration from $\left(t_{0}+k Q T\right)$ to $\left(t_{0}+(k+1) Q T-\right.$ $T / M$ ), associated with the symbol $\mathbf{x}(k)$. For $L \ll Q$ (which is plausible in several OFDM applications), the energy of the ISI samples is negligible compared to that of the non-ISI affected samples. Hence, we process only the ISI-free samples for channel estimation, i.e., the $M(Q-L)$ samples over the interval $\left(t_{0}+(k Q+L) T\right)$ to $\left(t_{0}+(k+1) Q T-T / M\right)$; correspondingly, $r_{i}^{(m)}$ s for $i=k Q+L, \ldots,(k+1) Q-1$ where $m=0, \ldots, M-1$ denotes the $m$ th sampling phase. Thus, the received signal for the $m$ th sampling phase corresponding to transmitted symbol $\mathbf{x}(k)$ is given by (9) at the bottom of the next page. Stacking all $M \mathbf{r}^{(m)}(k)(m=0, \ldots, M-1)$ vectors yields

$$
\begin{aligned}
\mathbf{r}(k) & =\underbrace{[0) T}_{\mathcal{A}}(k), \ldots, \mathbf{r}^{(M-1) T}(k)]^{T} \\
& =\underbrace{\left[\begin{array}{c}
\mathbf{H}^{(0)} \\
\vdots \\
\mathbf{H}^{(M-1)}
\end{array}\right]}_{\mathcal{H}} \mathbf{W}_{Q} \mathbf{s}(k)+\mathbf{n}(k) \\
& =\underbrace{\mathcal{A}(k)}_{\mathcal{H} \mathbf{s}(k)+\mathbf{n}(k) .}
\end{aligned}
$$

The multichannel model (10) for OFDM without $\mathrm{CP}$ yields an equivalent filtering matrix $\mathcal{A}$ of dimension $M(Q-L) \times Q$.

\section{B. Multichannel Model for Multiple Sensors}

Instead of time oversampling, multiple receive sensors each sampled at rate $1 / T$ also produce a multichannel signal model. However, in this situation, the signal $x(t)$ passes through different propagation channels and is received at an array of $M$ sensors. Again assuming $[0, L T]$ support for all composite channels, we obtain $h^{(m)}(l)=h^{(m)}\left(t_{0}+l T\right)$, and $\mathbf{h}^{(m)}=\left[h^{(m)}(0), h^{(m)}(1), \ldots, h^{(m)}(L)\right]^{T}$ as the discrete time equivalent channel impulse responses seen by the $m$ th sensor. As previously, collecting only the non ISI corrupted OFDM 


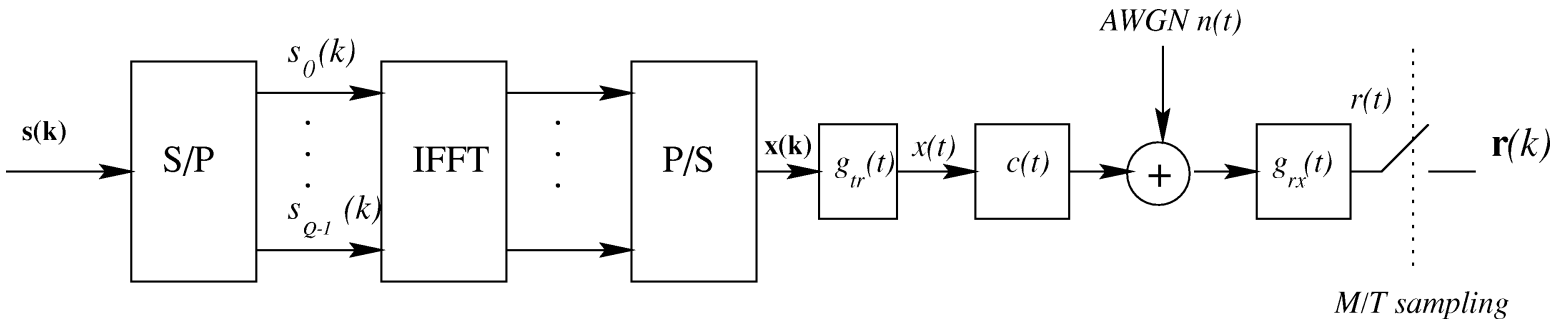

Fig. 1. Baseband OFDM system model.

symbols at each sensor and stacking leads to a multichannel signal model similar to (10).

Remarks:

1) Comparing (10) to the signal model in [1], Fig. 1 is analogous to a single carrier transmission of $\mathbf{x}(k)$, where $\mathbf{x}(k)=\mathbf{W}_{Q} \mathbf{s}(k)$ in OFDM and $\mathbf{W}_{Q}$ is a unitary matrix. This suggests the application of subspace channel estimation approach developed in [1] for conventional single carrier system for an OFDM system.

2) The multichannel signal model is overdetermined when $(M-1) Q>M L$. When $Q \gg L$ as assumed here, $M=2$ typically suffices to satisfy the necessary condition for the subspace method and is assumed throughout the paper.

\section{Subspace Based Channel Estimation}

We now describe a subspace based channel estimator based on the structure of $\mathcal{A}$ shown in (10). The time index $k$ is omitted when there is no confusion.

\section{A. Sufficient Conditions for Identifiability}

From (10), $\mathcal{A}=\mathcal{H} \mathbf{W}_{Q}(M(Q-L) \times Q)$ where $\mathcal{H}$ has the same Toeplitz structure as the filtering matrix $\mathcal{H}_{N}$ in [1] and $\mathbf{W}_{Q}$ is unitary. A sufficient condition for channel identifiability follows as a corollary of Theorem 1 and 2 in [1]. if:

Theorem 1 [1]: $\mathcal{H}$ is full column rank, i.e., $\operatorname{rank}(\mathcal{H})=\mathcal{Q}$,

1) the polynomials $H^{(m)}(z) \stackrel{\text { def }}{=} \sum_{j=0}^{L} h_{j}^{(m)} z^{j}$ have no common zero;

2) $Q \geq L$; and

3) at least one polynomial $H^{(m)}(z)$ has degree $L$.

Since $\mathbf{W}_{Q}$ is unitary, this directly leads to $\operatorname{rank}(\mathcal{A})=$ $\operatorname{rank}\left(\mathcal{H} \mathbf{W}_{Q}\right)=\operatorname{rank}(\mathcal{H})$. Therefore, the above conditions for
$\mathcal{H}$ to be full column rank also guarantee that $\mathcal{A}$ is full column rank.

Assume the user's transmitted information symbols $s_{i}(k)$ s to be i.i.d., sequences with zero mean and variance $\sigma_{s}^{2}\left(\sigma_{s}^{2}\right.$ can be set to unity, without loss of generality). Also assume that Nyquist pulse shaping is employed. For multiple sensors situation, the elements of $\mathbf{n}(k)$ in (10) are always AWGN. Note that this is not true for oversampling with a single receiver in general, as increasing the oversampling factor leads to correlated noise samples. However, for oversampling factor of $M=2$, the components of $\mathbf{n}(k)$ are approximately AWGN, particularly for systems for small excess bandwidth. Note that the method presented is readily generalized for arbitrary but known colored noise covariance, as shown in [1, App. C]. Hence, after collecting $N_{b}$ signal vectors, we have

$$
\begin{aligned}
\mathbf{Y} & =\left[\mathbf{r}(1), \ldots, \mathbf{r}\left(N_{b}\right)\right] \\
& =\mathcal{A} \underbrace{\left[\mathbf{s}(1), \ldots, \mathbf{s}\left(N_{b}\right)\right]}_{\mathbf{S}}+\mathbf{N} \\
& =\mathbf{X}+\mathbf{N} .
\end{aligned}
$$

The singular value decomposition (SVD) on the unpurturbed received signal matrix $\mathbf{X}$ yields

$$
\mathbf{X}=\left[\begin{array}{ll}
\mathbf{U}_{s} & \mathbf{U}_{n}
\end{array}\right]\left[\begin{array}{ll}
\boldsymbol{\Sigma}_{s} & \\
& \mathbf{0}
\end{array}\right]\left[\begin{array}{c}
\mathbf{V}_{s}^{H} \\
\mathbf{V}_{n}^{H}
\end{array}\right]
$$

where $\left[\mathbf{U}_{s}, \mathbf{U}_{n}\right]$ is an $M(Q-L) \times M(Q-L)$ unitary matrix. The $Q$ columns of $\mathbf{U}_{s}$ span the signal subspace, while $M(Q-L)-Q$ column vectors of $\mathbf{U}_{n}$ span a subspace (known as the noise subspace as in practice the SVD is applied on the noise purturbed signal matrix $\mathbf{Y}$ ) orthogonal to the signal subspace. $\boldsymbol{\Sigma}_{s}=\operatorname{diag}\left(\lambda_{1}, \lambda_{2}, \ldots, \lambda_{Q}\right)$ is a diagonal matrix consisting of

$$
\begin{aligned}
& \mathbf{r}^{(m)}(k)=\left[r_{k Q+L}^{(m)}, \ldots, r_{k Q+Q-1}^{(m)}\right]^{T} \\
& =\underbrace{\left[\begin{array}{cccccc}
h^{(m)}(L) & \cdots & h^{(m)}(0) & & & \\
& h^{(m)}(L) & \cdots & h^{(m)}(0) & & \\
& & \ddots & & \ddots & \\
& & & h^{(m)}(L) & \cdots & h^{(m)}(0)
\end{array}\right]}_{\mathbf{H}^{(\mathbf{m})}} \underbrace{\left[\begin{array}{c}
x_{k Q} \\
\vdots \\
x_{k Q+Q-1}
\end{array}\right]}_{\mathbf{x}(k)}+\underbrace{\left[\begin{array}{c}
v_{k Q+L}^{(m)} \\
\vdots \\
v_{k Q+Q-1}^{(m)}
\end{array}\right]}_{\mathbf{n}^{(m)}(k)} \\
& =\mathbf{H}^{(\mathbf{m})} \mathbf{x}(k)+\mathbf{n}^{(m)}(k) \\
& =\mathbf{H}^{(\mathbf{m})} \mathbf{W}_{Q} \mathbf{s}(k)+\mathbf{n}^{(m)}(k)
\end{aligned}
$$


$Q$ significant singular values corresponding to the signal subspace. The orthogonality property between signal subspace and noise subspace asserts

$$
\mathbf{U}_{n}(i)^{H} \mathcal{A}=0(i=1, \ldots, M(Q-L)-Q)
$$

where $\mathbf{U}_{n}(i)$ is the $i$ th column of $\mathbf{U}_{n}$.

Denote the time-reversed version of $\mathbf{h}^{(m)}$ as $\overline{\mathbf{h}}^{(m)}=$ $\left[h^{(m)}(L), h^{(m)}(L-1), \ldots, h^{(m)}(0)\right]^{T}$. The uniqueness of the estimate of $\overline{\mathbf{h}}^{(0)}, \ldots, \overline{\mathbf{h}}^{(M-1)}$ based on the noise subspace can be obtained as corollary of Theorem 2 in [1].

Theorem 2 [1]: Let $\overline{\mathbf{h}}=\left[\overline{\mathbf{h}}^{(0) T}, \ldots, \overline{\mathbf{h}}^{(M-1) T}\right]^{T}$, and $\overline{\mathbf{h}}^{\prime}$ be a $M(L+1) \times 1$ vector distinct from $\overline{\mathbf{h}}$; filtering matrix $\mathcal{H}$ and $\mathcal{H}^{\prime}$ are constructed using $\overline{\mathbf{h}}$ and $\overline{\mathbf{h}}^{\prime}$, respectively. When $Q \geq L$, if $\operatorname{ran}\left(\mathcal{H}^{\prime}\right)=\operatorname{ran}(\mathcal{H})$, then $\overline{\mathbf{h}}^{\prime}=\alpha \overline{\mathbf{h}}$ where $\alpha$ is a scalar.

It is easy to show that $\operatorname{ran}(\mathcal{A})=\operatorname{ran}\left(\mathcal{A}^{\prime}\right)$ also gives uniqueness of the channel estimation $\overline{\mathbf{h}}$ from (13) for the OFDM case, where now $\mathcal{A}=\mathcal{H} \mathbf{W}_{Q}$ and $\mathcal{A}^{\prime}=\mathcal{H}^{\prime} \mathbf{W}_{Q}$ are constructed using $\mathbf{h}$ and $\mathbf{h}^{\prime}$, respectively. Since multiplying by unitary matrix does not change the range of $\mathcal{H}$, therefore, if $\operatorname{ran}(\mathcal{A})=\operatorname{ran}\left(\mathcal{A}^{\prime}\right)$, then $\operatorname{ran}\left(\mathcal{H}^{\prime}\right)=\operatorname{ran}(\mathcal{H})$, and consequently $\overline{\mathbf{h}}^{\prime}=\alpha \overline{\mathbf{h}}$.

In summary, the sufficient condition for channel identifiability in the OFDM system of interest is as follows:

1) the polynomials $H^{(m)}(z) \stackrel{\text { def }}{=} \sum_{j=0}^{L} h_{j}^{(m)} z^{j}$ have no common zero;

2) $Q \geq L$

3) at least one polynomial $H^{(m)}(z)$ has degree $L$.

\section{Remarks:}

1) The application of noise subspace method in the OFDM of interest is a special case of [1]. Note that the requirement $Q \geq L$ is generally satisfied in practice for typical OFDM system and channel delay spreads.

2) Comparing to the other noise subspace method [3] which is not sensitive to channel order overestimation, the proposed method requires a good estimate on the channel order $L$.

\section{B. Blind Channel Estimator}

\section{Let}

$$
\begin{aligned}
\mathbf{U}_{n}(i)=\left[u_{i}(0)\right. & , \ldots, u_{i}(Q-L), \ldots, u_{i}((M-1) \\
& \left.\times(Q-L)), \ldots, u_{i}(M Q-M L-1)\right]^{T} .
\end{aligned}
$$

Exploiting the special structure of $\mathcal{H}$ yields

$$
\mathbf{U}_{n}(i)^{T} \mathcal{H}=\overline{\mathbf{h}}^{T} \mathcal{U}_{i}
$$

where the $M(L+1) \times Q$ dimension matrix $\mathcal{U}_{i}=$ $\left[\mathcal{U}_{i}^{(0) T}, \ldots, \mathcal{U}_{i}^{(M-1) T}\right]^{T}$ is generated from vector $\mathbf{U}_{n}(i)$, and each $(L+1) \times Q$ submatrix $\mathcal{U}_{i}^{(m)}(m=0, \ldots, M-1)$ is formed as shown in the equation at the bottom of the page. When only an estimate of the noise subspace $\hat{\mathbf{U}}_{n}$ is available in practice, (13) suggests the channel estimator

$$
\hat{\overline{\mathbf{h}}}=\arg \min _{\|\overline{\mathbf{h}}\|=1} \sum_{i=1}^{M(Q-L)-Q}\left\|\hat{\mathbf{U}}_{n}(i)^{H} \mathcal{A}\right\|^{2} .
$$

But from (15)

$$
\begin{aligned}
\left\|\mathbf{U}_{n}(i)^{H} \mathcal{A}\right\|^{2} & =\mathbf{U}_{n}(i)^{H} \mathcal{H} \mathbf{W}_{Q} \mathbf{W}_{Q}^{H} \mathcal{H}^{H} \mathbf{U}_{n}(i) \\
& =\overline{\mathbf{h}}^{T}\left(\mathcal{U}_{i}\right)^{*} \mathcal{U}_{i}^{T} \overline{\mathbf{h}}^{*}
\end{aligned}
$$

Thus, by defining $\tilde{\mathbf{h}}=(\overline{\mathbf{h}})^{*}$, and

$$
\mathcal{G}=\left[\mathcal{U}_{1}, \ldots, \mathcal{U}_{M(Q-L)-Q}\right]^{*}
$$

the channel information $\tilde{\mathbf{h}}$ is determined by

$$
\hat{\tilde{\mathbf{h}}}=\arg \min _{\|\tilde{\mathbf{h}}\|=1} \tilde{\mathbf{h}}^{H} \hat{\mathcal{G}} \hat{\mathcal{G}}^{H} \tilde{\mathbf{h}}
$$

where $\hat{\mathcal{G}}$ is the estimate of $\mathcal{G}$. It is well known that $\tilde{\mathbf{h}}$ (or equivalently $\mathbf{h}$ ) is the eigenvector corresponding to the smallest eigenvalue of the matrix $\hat{\mathcal{G}} \hat{\mathcal{G}}^{H}$.

\section{Performance Analysis}

A first-order performance analysis is conducted on the proposed estimator to estimate the mean square error (MSE) at high signal-to-noise ratio (SNR) similar to that adopted for DS-CDMA systems in [12].

Theorem 3: Assuming that both noise and the signals are zero mean i.i.d. random variables with variance $\sigma_{n}^{2}$ and $\sigma_{s}^{2}$, respectively, the MSE of the channel vector estimate $\hat{\mathbf{h}}$ from (19) is approximated by

$$
E\left(\|\hat{\tilde{\mathbf{h}}}-\tilde{\mathbf{h}}\|^{2}\right) \approx \frac{\sigma_{n}^{2}\left\|\mathcal{G}^{+}\right\|^{2}}{\sigma_{s}^{2} N_{b}} .
$$

Proof: See the Appendix.

The closed form MSE expression (20) is compact and enables us to study the estimator's performance dependence on the key system parameters - such as the input SNR, the length of data record $N_{b}$, and the number of subcarriers $Q$. As expected, the MSE decreases with increasing input SNR and $N_{b}$. Although the dependence on $Q$ is implicit, its effect can be investigated numerically. Intuitively, increasing $Q$ gives rise to larger noise subspace dimension and more constraints on the channel vector-resulting in improved estimator accuracy. This is later verified by simulation example in Section IV.

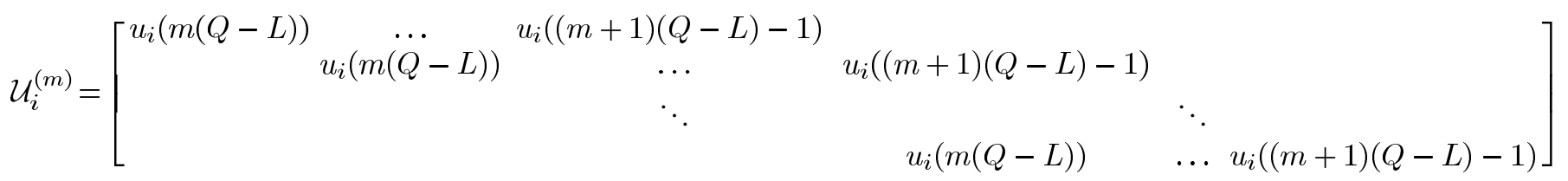




\section{Simulation Results}

Monte Carlo simulations are conducted to assess the effectiveness of the proposed blind estimator with comparison to other two reported subspace channel estimation methods for OFDM [2], [3]. The proposed method eliminates the CP, but requires multiple sensors or oversampling at the receiver; we assume $M=2$. The methods of [2], [3] used as comparison baselines require $\mathrm{CP}$; thus, results from them are obtained based on one sensor and usual sampling rate (rate $1 / \mathrm{T}$; i.e., no oversampling) with the length of the CP set to a quarter of the available sub carriers $Q$, as in [2] and [3].-

To evaluate the estimation error, the normalized root meansquared error (RMSE)

$$
\operatorname{RMSE}=\frac{1}{\|\mathbf{h}\|} \sqrt{\frac{1}{N_{m} M(L+1)} \sum_{p=1}^{N_{m}}\left\|\hat{\mathbf{h}}_{p}-\mathbf{h}\right\|^{2}}
$$

and the average bias, defined as

$$
\text { BIAS }=\frac{1}{N_{m} M(L+1)} \sum_{k=0}^{M-1} \sum_{l=0}^{L}\left|\sum_{p=1}^{N_{m}} \hat{h}_{p}^{(k)}(l)-h^{(k)}(l)\right|
$$

are used, where the subscript $p$ refers to the $p$ th simulation run and $N_{m}$ denotes the number of runs. Information sequence $s_{i}(k) \mathrm{s}$ are BPSK modulated. Input SNR is defined as $\operatorname{SNR} \stackrel{\text { def }}{=} 10 \log _{10}\left(\sigma_{s}^{2} / \sigma_{n}^{2}\right)$. Two time-invariant multipath channel setups, with order $L=3$ and $L=4$, respectively, are generated according to Hoeher's method [13] by setting maximum Doppler shift to zero. The channel coefficients are listed below.

1) Setup A $(L=3)$ :

$$
\begin{aligned}
\mathbf{h}^{(1)}= & {[(-0.1892,0.4273),(-0.2839,0.6984),} \\
& (0.1274,0.4321),(-0.0451,0.0912)]^{T} \\
\mathbf{h}^{(2)}= & {[(0.3600,0.1388),(0.1041,0.4126),} \\
& (0.0914,0.1885),(0.2052,-0.0739)]^{T} .
\end{aligned}
$$

2) Setup B $(L=4)$ :

$$
\begin{aligned}
\mathbf{h}^{(1)}= & {[(0.3825,0.0010),(0.5117,0.2478),(-0.3621,0.3320),} \\
& (-0.4106,0.3428),(0.0087,0.0546)]^{T} \\
\mathbf{h}^{(2)}= & {[(-0.2328,0.1332),(-0.3780,-0.3794),} \\
& (-0.0320,-0.4532),(0.5081,-0.0125), \\
& (0.4195,0.0220)]^{T} .
\end{aligned}
$$

Note that there is a complex scalar ambiguity inherent in the blind channel estimator (as described in Theorem 2 in Section III). During the simulations, the amplitude ambiguity is handled by assuming the true channel vector $\mathbf{h}$ to unit norm and similarly normalizing the estimate. Without further processing, the phase ambiguity cannot be resolved. In our work, this phase ambiguity is determined from phase $\left(h^{(1)}(0) / \hat{h}^{(1)}(0)\right)$ and used to compensate the channel estimate prior to the sample MSE computations. The results shown are averaged over $\mathbf{h}^{(1)}$ and $\mathbf{h}^{(2)}$

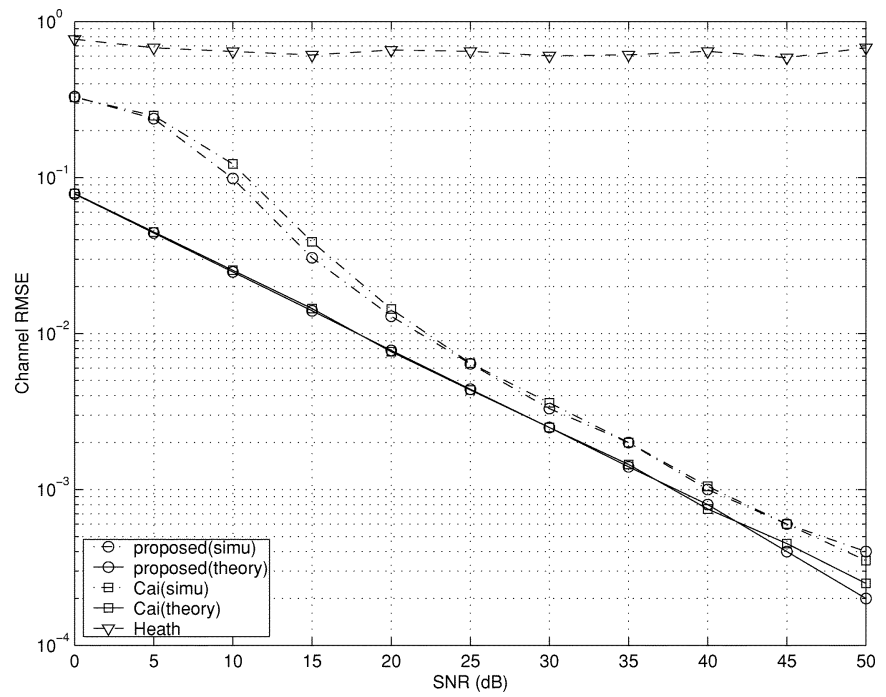

(a)

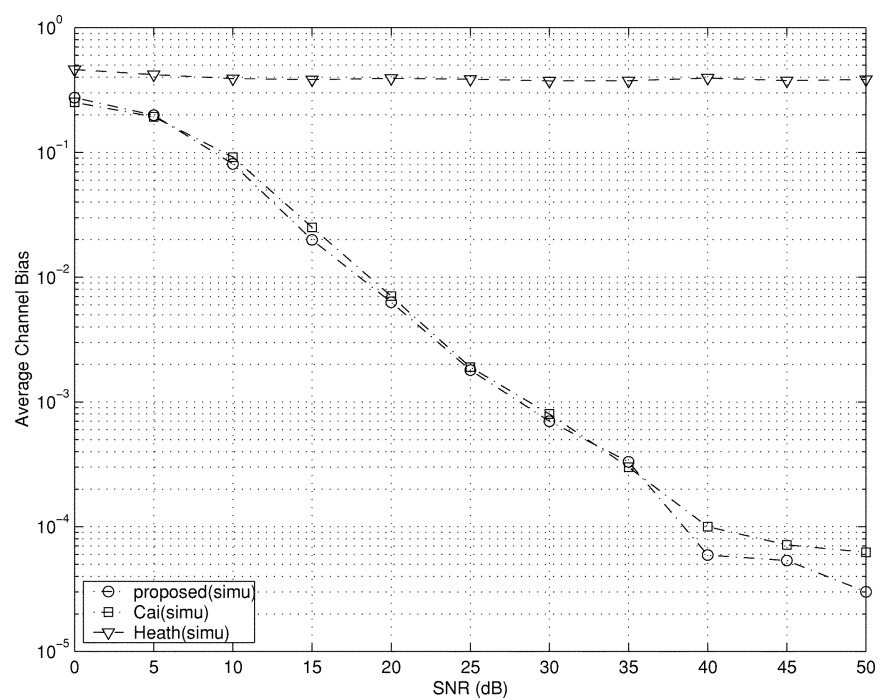

(b)

Fig. 2. Channel error versus SNR.

(when setup A or B is assumed) for all three methods. 100 runs are carried out to obtain the average, i.e., $N_{m}=100$.

Example 1: In this example, we examine the estimator error as a function of the input SNRs and compare it with the results from other two CP-based methods, using the following setup ${ }^{1}$ : $Q=15, N_{b}=120$ and channel setup A. It can be seen that both our approach and the method in [3] (marked as Cai) perform much better than that of [2] (marked as Heath), reflecting the fast convergence property of the noise subspace estimator for small data record. Also note that the estimator error of our proposed method is close to the Cai method for comparable computation complexity.

In addition, the MSE results evaluated via (20) for both the proposed method and [3] $]^{2}$ are plotted together in Fig. 2(a) to verify the perturbation analysis in Section III-C. Solid line stands for the theoretical result and dash line is the simulation

\footnotetext{
${ }^{1}$ For the CP-based methods, the length of the $\mathrm{CP}$ is set to four in this example.

${ }^{2}$ For this method, the MSE of the channel estimate can be derived in the same lines; the expression is very similar to (20).
} 


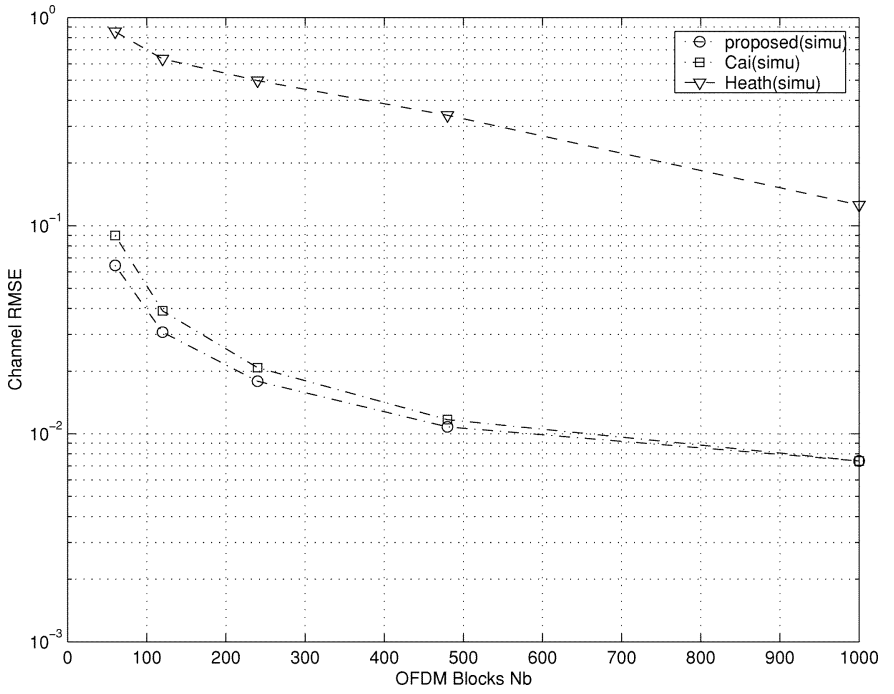

(a)

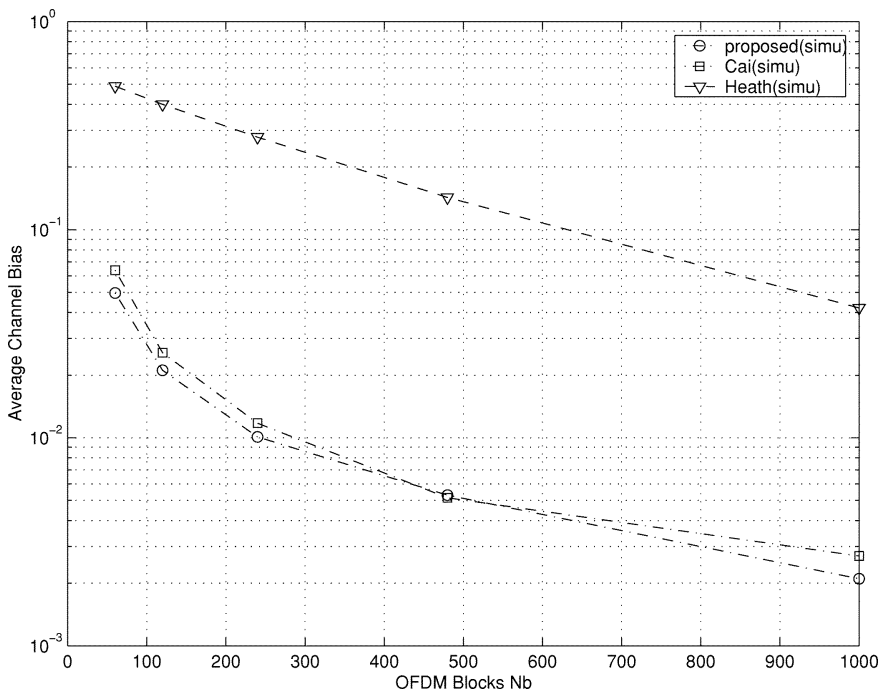

(b)

Fig. 3. Channel error versus number of data blocks $N_{b}$ (simulation results, $\mathrm{SNR}=15 \mathrm{~dB}$ )

result. It shows good agreement of MSEs (when SNR $\geq 20 \mathrm{~dB}$ ) obtained from simulation and (20).

Example 2: In the second example, with the same $Q$ and the same channel setup as before, we illustrate the estimator error as a function of the number of data blocks $N_{b}$. For SNR $=15 \mathrm{~dB}$, Fig. 3 shows that the estimation accuracy improves as the number of data blocks increases for all three subspace methods. Note that the noise subspace methods (both ours and Cai's) achieve low estimate error $(<0.1)$ with only 60 OFDM blocks, while the spectrum fitting method (Heath's) requires more than 1000 OFDM blocks for comparable performance. The superior performance of the noise subspace method over the spectrum fitting method makes them a possible candidate for wideband communication scenarios where the channel is time-invariant for only a few OFDM symbols. Moreover, the proposed method avoids the $\mathrm{CP}$ and, therefore, leads to higher throughput than [3].

Example 3: In this example, simulation results for both channel setup A (solid line) and B (dash line) for all three

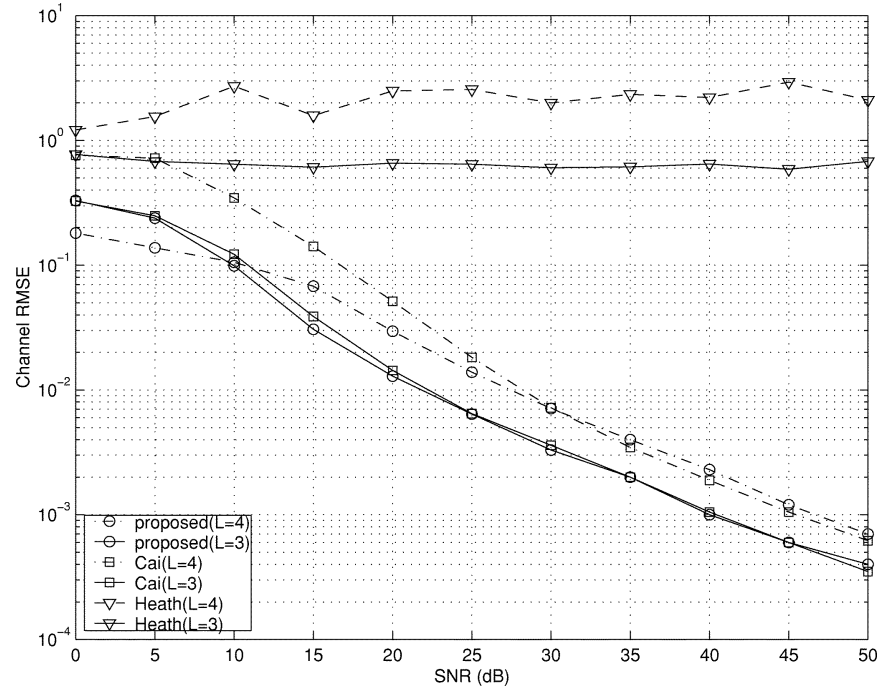

(a)

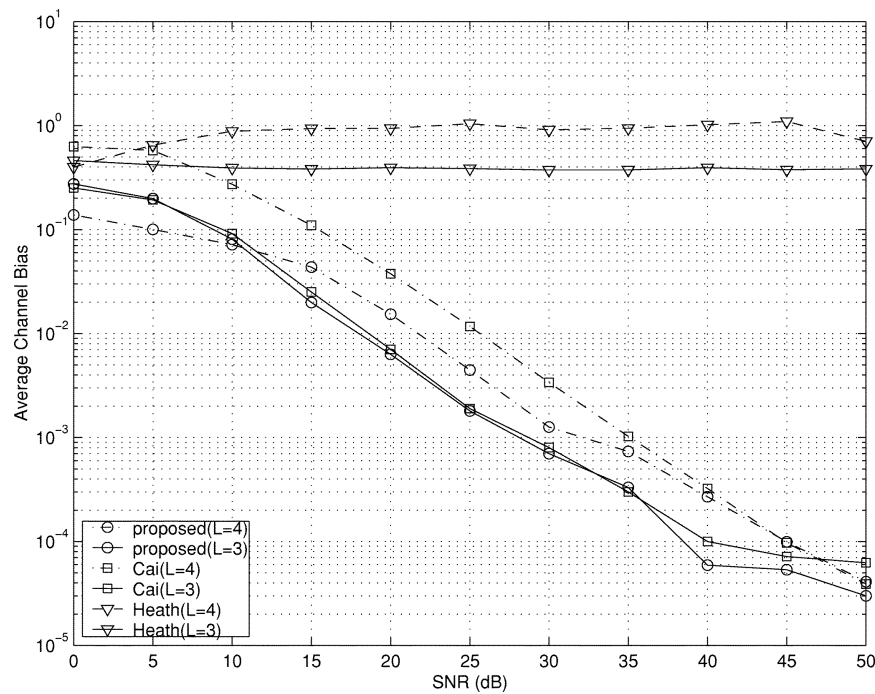

(b)

Fig. 4. Channel error for different channel order $L$ (simulation results).

methods are drawn in the same figure (see Fig. 4) to highlight the estimate error for different channel orders. System parameters $Q, N_{b}$ are the same as example 1 . As expected, for all methods, estimator performance degrades for longer channel. Note the proposed method performs better than [3] at SNR level lower than $25 \mathrm{~dB}$. But as SNR increases, the performance gap between these two methods converges.

Example 4: Finally, the effect of varying $Q$ (meaning longer OFDM symbol duration) on the estimator error for the two noise-subspace methods (proposed and Cai) is investigated in Fig. 5 with $\mathrm{SNR}=40 \mathrm{~dB}, N_{b}=2000$, channel setup A, and $Q$ varying from 15 to 47 (the length of the $\mathrm{CP}$ is set to $(Q+1) / 4$ and changed accordingly for Cai's method). Larger $Q$ means larger dimension of the noise subspace $((M-1) Q-L$ for the proposed method and $(M Q / 4)-L$ for Cai's), yielding more constraints on the channel vector [as in (13)] and, thus, leads to improvement in the channel estimate. Also note that for the proposed method, the noise subspace dimension increases faster with $Q$ than it does for Cai's method, leading to larger performance improvement. 


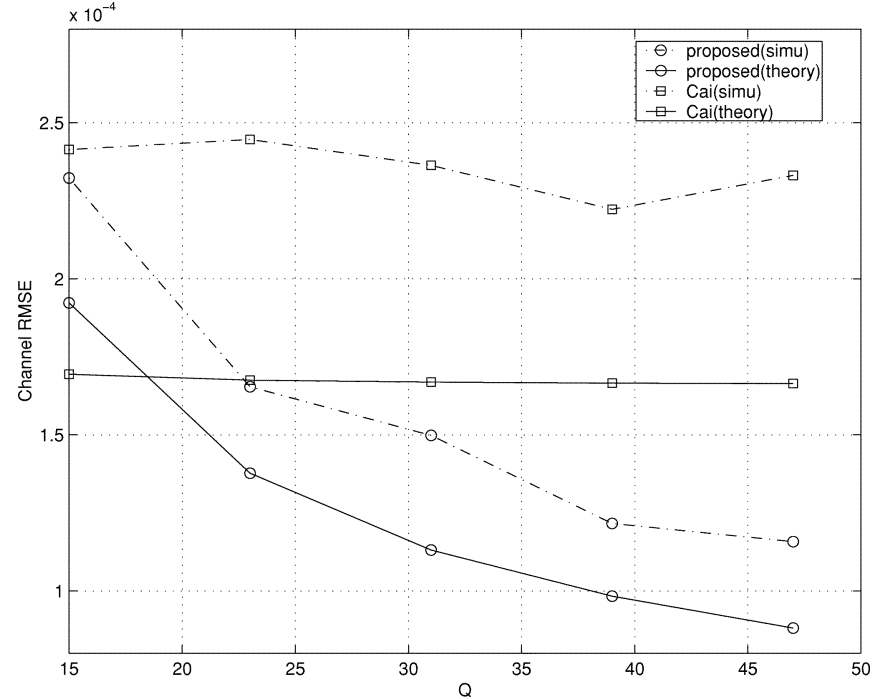

Fig. 5. RMSE versus $Q$.

\section{CONCLUSION}

In this paper, we presented a subspace based blind channel estimator for OFDM system without the CP. A sufficient condition on identifiability was also developed, along with a first-order performance analysis on the channel estimate. The algorithm is attractive for its potential to increase the system's channel utilization due to the elimination of the CP. Comparison of the proposed method with other two reported subspace channel estimation methods by computer simulations illustrates the superior performance of the proposed method with regard to both the estimate accuracy and the speed of convergence.

\section{APPENDIX \\ ASYMPTOTIC CHANNEL ESTIMATION MSE}

An approximation for the channel estimate's MSE for high sample SNR and/or large sample size is obtained based on the first-order perturbation theory given in [14].

Lemma 1 [14]: Assuming X permit the SVD

$$
\mathbf{X}=\left[\begin{array}{ll}
\mathbf{U}_{s} & \mathbf{U}_{n}
\end{array}\right]\left[\begin{array}{ll}
\boldsymbol{\Sigma}_{s} & \\
& \mathbf{0}
\end{array}\right]\left[\begin{array}{c}
\mathbf{V}_{s}^{H} \\
\mathbf{V}_{n}^{H}
\end{array}\right]
$$

the first-order approximation of the perturbation to the subspace $\mathbf{U}_{n}$ due to additive perturbation $\mathbf{N}$ to $\mathbf{X}$ is

$$
\Delta \mathbf{U}_{n}=-\mathbf{U}_{s} \Sigma_{s}^{-1} \mathbf{V}_{s}^{H} \mathbf{N}^{H} \mathbf{U}_{n}=-\mathbf{X}^{+} \mathbf{N}^{H} \mathbf{U}_{n} .
$$

For the problem under consideration, the received signal matrix $\mathbf{X}$ is perturbed by AWGN noise $\mathbf{N}$. Following the above lemma, the perturbation on the noise subspace is, therefore, $\Delta \mathbf{U}_{n}=-\mathbf{X}^{+} \mathbf{N}^{H} \mathbf{U}_{n}$. Since $\mathcal{G}$ is constructed from $\mathbf{U}_{n}$ [see
(18) and (15)], the perturbation $\Delta \mathcal{G}$ to $\mathcal{G}$ is additive and can be formed in the same way from $\Delta \mathbf{U}_{n}$. In addition, the structure of $\Delta \mathcal{G}$ gives

$$
\begin{aligned}
\Delta \mathcal{G}^{H} \tilde{\mathbf{h}} & =\left[\begin{array}{c}
\Delta \mathcal{U}_{1} \tilde{\mathbf{h}} \\
\vdots \\
\Delta \mathcal{U}_{M(Q-L)-Q} \tilde{\mathbf{h}}
\end{array}\right] \\
& =\left[\begin{array}{c}
\mathcal{H}^{H} \Delta \mathbf{U}_{n}(1) \\
\vdots \\
\mathcal{H}^{H} \Delta \mathbf{U}_{n}(M(Q-L)-Q)
\end{array}\right]
\end{aligned}
$$

where $\Delta \mathbf{U}_{n}(i)$ is the $i$ th column of $\Delta \mathbf{U}_{n}$. Note that the estimator (19) suggests the channel vector $\tilde{h}$ is the unit null vector of $\mathcal{G}$ when there is no noise. By applying the lemma again, the above observation immediately yields the perturbation of the channel estimate

$$
\Delta \tilde{\mathbf{h}}=-\mathcal{G}^{+} \Delta \mathcal{G}^{H} \tilde{\mathbf{h}}
$$

where $\mathcal{G}^{+}$is generated from $\mathcal{G}_{\mathrm{s}}$ singular values and its left/right singular vectors in a similar way to $\mathbf{X}^{+}$in (23). Substituting (24) and (23) into the above perturbation leads to (26) at the bottom of the page. Next, before computing the channel estimate MSE, we prove the following lemma.

Lemma 2: Assume $N$ is an $m \times n$ matrix where each element is zero-mean i.i.d. random variable with variance $\sigma^{2}$. Also assume $J$ is an $m \times m$ deterministic matrix. Then $E\left(N^{H} J N\right)=$ $\sigma^{2} \operatorname{trace}(J) I_{n}$ where $I_{n}$ is an $n \times n$ identity matrix and trace $(\cdot)$ gives the trace of the matrix.

Proof: Let $(\cdot)_{i j}$ be the element in the $i$ th row and $j$ th column of matrix $(\cdot)$. Define $F=N^{H} J N$. Then

$$
\begin{aligned}
E\left(F_{i j}\right) & =E\left(\sum_{l=1}^{m}\left[\left(\sum_{k=1}^{m} N_{k i}^{*} \cdot J_{k l}\right) \cdot N_{l j}\right]\right) \\
& =\sum_{l=1}^{m}\left[\sum_{k=1}^{m} J_{k l} \cdot E\left(N_{k i}^{*} \cdot N_{l j}\right)\right] \\
& = \begin{cases}\sum_{l=1}^{m} J_{l l} \sigma^{2}, & i=j \\
0, & \text { otherwise. }\end{cases}
\end{aligned}
$$

Therefore, $E\left(N^{H} J N\right)=\sigma^{2} \sum_{l=1}^{m} J_{l l} I_{n}=\sigma^{2} \operatorname{trace}(J) I_{n}$.

By using Lemma 2, it is easy to see that for the considered problem, we have

$$
\begin{aligned}
E\left(\mathbf{N}^{H} \mathbf{U}_{n}(i) \mathbf{U}_{n}^{H}(j) \mathbf{N}\right) & =\sigma_{n}^{2} \operatorname{trace}\left(\mathbf{U}_{n}(i) \mathbf{U}_{n}^{H}(j)\right) I \\
& =\sigma_{n}^{2} \delta(i-j) I
\end{aligned}
$$

with $\delta(\cdot)$ being the Kronecker Delta function and, consequently

$$
E\left(\mathbf{d d}^{H}\right)=\sigma_{n}^{2} I .
$$

$$
\Delta \tilde{\mathbf{h}}=\mathcal{G}^{+}\left[\begin{array}{c}
\mathcal{H}^{H} \mathbf{X}^{+} \mathbf{N}^{H} \mathbf{U}_{n}(1) \\
\vdots \\
\mathcal{H}^{H} \mathbf{X}^{+} \mathbf{N}^{H} \mathbf{U}_{n}(M(Q-L)-Q)
\end{array}\right]=\mathcal{G}^{+} \underbrace{\left[\begin{array}{ccc}
\mathcal{H}^{H} \mathbf{X}^{+} & & \\
& \ddots & \\
& & \mathcal{H}^{H} \mathbf{X}^{+}
\end{array}\right]}_{\mathbf{B}} \underbrace{\left[\begin{array}{c}
\mathbf{N}^{H} \mathbf{U}_{n}(1) \\
\vdots \\
\mathbf{N}^{H} \mathbf{U}_{n}(M(Q-L)-Q)
\end{array}\right]}_{\mathbf{d}}
$$


Hence, the MSE of the channel estimate is

$$
\begin{aligned}
E\left(\|\Delta \tilde{\mathbf{h}}\|^{2}\right) & =E\left[\operatorname{trace}\left(\Delta \tilde{\mathbf{h}} \Delta \tilde{\mathbf{h}}^{H}\right)\right] \\
& =\operatorname{trace}\left(E\left(\mathcal{G}^{+} \mathbf{B} \mathbf{d} \mathbf{d}^{H} \mathbf{B}^{H} \mathcal{G}^{+H}\right)\right) \\
& =\operatorname{trace}\left(\mathcal{G}^{+} \mathbf{B} E\left(\mathbf{d d}^{H}\right) \mathbf{B}^{H} \mathcal{G}^{+H}\right) \\
& =\sigma_{n}^{2} \cdot \operatorname{trace}\left(\mathcal{G}^{+} \mathbf{B} \mathbf{B}^{H} \mathcal{G}^{+H}\right) .
\end{aligned}
$$

In addition, it is shown in [14] that $\mathcal{A}^{H} \mathbf{X}^{+} \mathbf{X}^{+H} \mathcal{A}=\hat{\mathbf{R}}_{S}^{-1}$ where $\hat{\mathbf{R}}_{S} \stackrel{\text { def }}{=} \mathbf{S S}^{H}$ is the "estimated" data covariance. For large $N_{b}$ (number of data blocks), approximation $\hat{\mathbf{R}}_{S}^{-1} \approx\left(1 / N_{b}\right) \mathbf{R}_{S}^{-1}=\left(1 / N_{b} \sigma_{s}^{2}\right) I$ is reasonable. Thus

$$
\begin{aligned}
E\left(\|\Delta \tilde{\mathbf{h}}\|^{2}\right) & \approx \frac{\sigma_{n}^{2} \cdot \operatorname{trace}\left(\mathcal{G}^{+} \mathcal{G}^{+H}\right)}{N_{b} \sigma_{s}^{2}} \\
& =\frac{\sigma_{n}^{2}\left\|\mathcal{G}^{+}\right\|^{2}}{\sigma_{s}^{2} N_{b}}
\end{aligned}
$$

\section{ACKNOWLEDGMENT}

The authors would like to thank the associate editor and anonymous reviewers for their constructive comments that improved the quality of this paper. Fruitful discussions with Mr. H. Yan is also gratefully appreciated. The authors would also like to acknowledge Dr. R. W. Heath, Jr., for providing the code for spectrum fitting based blind channel estimation in [2].

\section{REFERENCES}

[1] E. Moulines, P. Duhamel, J. Cardoso, and S. Mayrargue, "Subspace methods for the blind identification of multichannel FIR filters," IEEE Trans. Signal Processing, vol. 43, pp. 516-525, Feb. 1995.

[2] R. W. Heath and G. B. Giannakis, "Exploiting input cyclostationarity for blind channel identification in OFDM systems," IEEE Trans. Signal Processing, vol. 47, pp. 848-856, Mar. 1999.

[3] X. Cai and A. N. Akansu, "A subspace method for blind channel identification in OFDM systems," in Proc. ICC2000, 2000, pp. 929-933.

[4] J. A. C. Bingham, "Multicarrier modulations for data transmission: An idea whose time has come," IEEE Commun. Mag., vol. 28, pp. 5-14, May 1990.

[5] W. Y. Zou and Y. Wu, "COFDM: An overview," IEEE Trans. Broadcasting, vol. 41, pp. 1-8, Mar. 1995.

[6] J. A. C. Bingham, ADSL, VDSL and Multicarrier Modulation. New York: Wiley, 2000.

[7] Wireless LAN Medium Access Control (MAC) and Physical Layer (PHY) specifications: High speed physical layer in the 5 GHZ band, IEEE Standard 802.11a, 1999.
[8] H. Sari, G. Karam, and I. Jeanclaude, "Transmission techniques for digital terrestrial TV broadcasting," IEEE Commun. Mag., vol. 33, pp. 100-109, Feb. 1995.

[9] O. Edfors, M. Sandell, J. van de Beek, S. Kate, and P. O. Borjesson, "OFDM channel estimation by singular value decomposition," IEEE Trans. Commun., vol. 46, pp. 931-939, July 1998.

[10] Y. Li, L. J. Cimini Jr., and N. R. Sollenberger, "Robust channel estimation for OFDM systems with rapid dispersive fading channels," IEEE Trans. Commun., vol. 46, pp. 902-915, July 1998.

[11] Y. Li and N. R. Sollenberger, "Adaptive antenna arrays for OFDM systems with co-channel interference," IEEE Trans. Commun., vol. 47, pp. 217-229, Feb. 1999.

[12] H. Liu and G. Xu, "A subspace method for signature waveform estimation in synchronous CDMA systems," IEEE Trans. Commun., vol. 44, pp. 1346-1354, Oct. 1996.

[13] P. Hoeher, "A statistical discrete-time model for the WSSUS multipath channel," IEEE Trans. Veh. Technol., vol. 41, pp. 461-468, Nov. 1992.

[14] F. Li, H. Liu, and R. J. Vaccaro, "Performance analysis for DOA estimation algorithms: Further unification, simplification, and observations," IEEE Trans. Aerosp., Electron. Syst., vol. 29, pp. 1170-1184, Oct. 1993.

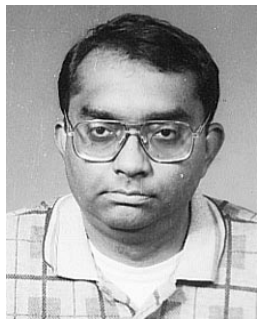

Sumit Roy (S'84-M'88-SM'00) received the B.Tech. degree from the Indian Institute of Technology, Kanpur, in 1983, the M.S. and Ph.D. degrees from the University of California, Santa Barbara, in 1985 and 1988, respectively, all in electrical engineering, and the M.A. degree in Statistics and Applied Probability, in 1988. His previous academic appointments were at the Moore School of Electrical Engineering, University of Pennsylvania, Philadelphia, and at the University of Texas, San Antonio.

He is presently an Associate Professor of Electrical Engineering at the University of Washington, Seattle. His research interests include analysis/design of communication systems/networks, with a topical emphasis on next generation mobile/wireless networks.

Dr. Roy is a member of several technical committees for the IEEE Communications Society, and is an Editor for the IEEE TRANSACTIONS ON COMMUNICATIONS and IEEE TRANSACTIONS ON WIRELESS COMMUNICATIONS.

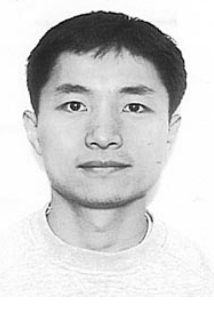

Chengyang Li received the B.S. and M.S. degrees from the University of Electronic Science and Technology, Chengdu, China, in 1992 and 1995, respectively, all in electrical engineering. He is currently working toward the Ph.D. degree at the University of Washington, Seattle.

Since 1999, he has been a Research Assistant at the FUndamentals of Networking LABoratory (FUNLAB), University of Washington. His research interests include OFDM/Multicarrier CDMA systems, multiuser communications, and wireless LAN. 